\title{
Ultrasound-guided fine-needle aspiration cytology: Diagnostic value in breast solid masses.
}

\author{
Gustavo Febles, Andrés Dell'Acqua, Andrea Cristiani, Enrique Folle, Álvaro Vázquez \\ Breast Center for Diagnosis and Treatment (CENDYTMA), Spanish Association, Montevideo, Uruguay.
}

\begin{abstract}
Objective: To evaluate the efficacy of ultrasound-guided fine-needle aspiration cytology for the diagnosis of breast solid masses.

Materials and method: It is a retrospective study with 705 patients carrying a solid breast mass who underwent ultrasound-guided fine-needle aspiration cytology for the diagnosis. The fine needle aspiration cytology (FNAC) was performed with ultrasound guidance and a $21 \mathrm{G}$ gauge needle was used. The cytological results were classified as benign, suspicious, malignant or insufficient. The benign results were considered negative for malignancy and the malignant and suspect results were considered positive for malignancy. Insufficient results were not included in analysis of the accuracy of the study.
\end{abstract}

In all cases the histological result of the masses was obtained and it was taken as reference test. The ultrasound-guided FNAC accuracy was evaluated for all cases and then specifically for the masses classified in the categories 3,4 and 5 of BI-RADS.

Results: When all cases were included the results were the following: sensitivity $96 \%$, specificity $92 \%$, positive predictive value $97 \%$, negative predictive value $89 \%$, false positives $3 \%$, false negatives $11 \%$ and overall accuracy $95 \%$. For the BI-RADS category 3 the results were the following: sensitivity $100 \%$, specificity $98 \%$, positive predictive value $90 \%$, negative predictive value $100 \%$, false positives $10 \%$, there were no false negatives and overall accuracy $98 \%$.

For the BI-RADS category 4 the results were the following: sensitivity $92 \%$, specificity $85 \%$, positive predictive value $95 \%$, negative predictive value $78 \%$, false positives $5 \%$, false negatives $21 \%$ and overall accuracy $91 \%$. For the BI-RADS category 5 the results were the following: sensitivity $99 \%$, specificity $75 \%$, positive predictive value $99.6 \%$, negative predictive value $40 \%$, false positives $0.4 \%$, false negatives $60 \%$ and overall accuracy $99 \%$.

Conclusion: Ultrasound-guided FNAC is a valuable diagnostic technique for breast solid masses. In the probably benign masses (BI-RADS 3) its objective is to rule out malignancy for safely avoid biopsy and planning an imaging follow-up. In the suspicious and highly suggestive of malignancy masses (BI-RADS 4 and 5) the objective is to confirm malignancy prior to definitive treatment, when neoadjuvant chemotherapy is not needed.

Keywords: Breast, Fine-needle, Aspiration, Cytology, Cancer.

\section{Introduction}

Fine-needle aspiration cytology (FNAC) is a minimally invasive diagnostic procedure that can be used in palpable and non-palpable breast lesions. In case of non-palpable breast lesions, the procedure is done under stereotactic or ultrasound guidance. The diagnosis is based on the analysis of the characteristics of single cells contained in the samples of the lesion.
The role of FNAC has been challenged with the development of percutaneous techniques of histological diagnosis (core biopsy and vacuum-assisted biopsy). These techniques yield higher volume samples, preserving tissue structure of the lesion and are processed and analyzed as a histological sample. The diagnostic accuracy is higher and it is possible to analyze the molecular profiling of cancers diagnosed. For these reasons, many institutions have abandoned the 
use of FNAC in breast lesions, and currently are using percutaneous techniques of histological diagnosis.

However, FNAC remains as a reliable diagnostic method, if it is done with rigorous quality conditions in obtaining the sample and if there is a highly trained medical cytologist in the institution [1]. The method is cheaper than the histological diagnosis techniques and is accessible by institutions without a high technological development. Is a less traumatic procedure for the patient and the results may be available in less time.

FNAC and percutaneous techniques of histological diagnosis can coexist if there are protocols with the adequate indications of each technique. FNAC has its applications in the evacuation of cystic breast lesions, the diagnosis of breast masses, and the preoperative evaluation of suspicious axillary lymph nodes in patients with breast cancer already known.

We believe that in case of breast masses the objectives, reliability and usefulness of the FNAC are linked to the imaging features of the lesion. The aim of this work is to evaluate the efficacy of ultrasound-guided FNAC for the diagnosis of breast solid masses. The results also were correlated with the likelihood of malignancy of the lesions according to their imaging features.

\section{Materials and Methods}

It is a retrospective study where 705 consecutive patients carrying a solid breast mass were included. The work was done at the Breast Center for Diagnosis and Treatment (CENDYTMA) of the Spanish Association (Montevideo, Uruguay).

All patients were studied with mammography and breast ultrasound. Palpable and non-palpable masses were included in the analysis. The cystic masses were not included. The imaging features of the masses were analyzed and classified according to the BI-RADS categories (Breast Imaging Reporting and Data Base; American College of Radiology) [2]. The ultrasound equipment was a General Electric, Logiq P5 model, with a $10 \mathrm{MHz}$ linear transducer.

The fine needle aspiration cytology (FNAC) was performed with ultrasound guidance and a $21 \mathrm{G}$ gauge needle was used. The procedure was made with the patient's consent and in most cases the medical cytologist was present. Several passes were made through the lesion, with permanent aspiration. In all cases the sample was spread on a glass slide and allowed to air dry. May Grünwald Giemsa staining technique was used.

The cytological results were classified as benign, suspicious, malignant or insufficient. When the medical cytologist was present, a proof of sufficiency of the sample was performed. In cases with insufficient samples, the procedure was repeated at the time. When a diagnostic sample could not be obtained, the final result remained insufficient. For the purposes of the calculations, the benign results were considered negative for malignancy and the malignant and suspect results were considered positive for malignancy. Insufficient results were not included in analysis of the accuracy of the study.

In all cases the histological result of the masses was obtained, and it was taken as reference test. The ultrasoundguided FNAC accuracy was evaluated for all cases and then specifically for the masses classified in the categories 3, 4 and 5 of BI-RADS. Oval masses, with larger diameter parallel to the skin and circumscribed margins were classified as BI-RADS 2 (benign) if they had no changes in relation to previous studies and as BI-RADS 3 (probably benign) if we had no previous studies to compare.

Masses with not circumscribed margins (undefined, microlobulated or angular) or with suspicious microcalcifications inside, were classified as BI-RADS 4 (suspicious). Nodules with irregular morphology and spiculated margins were classified as BI-RADS 5 (highly suggestive of malignancy). For the calculations the SPSS version 12.0 software was used.

\section{Results}

The average age of patients studied was 60 years (19-92 years). 2 nodules were classified as BI-RADS $2(0.3 \%)$, 111 were classified as BI-RADS $3(16 \%), 317$ were classified as BI-RADS $4(45 \%)$ and 275 were classified BIRADS 5 (39\%). In 177 cases (25\%) the cytological result was negative for malignancy and in 505 cases $(72 \%)$ was positive for malignancy. The sample was insufficient in 23 cases $(3 \%)$ of which 17 were categorized as BI-RADS 4 and the other 6 as BI-RADS 5.

The final histological result obtained by percutaneous biopsy or surgical biopsy was benign in 179 cases $(25 \%)$ and malignant in 526 cases $(75 \%)$. The average size of diagnosed malignant tumors was $18 \mathrm{~mm}$ ( 1 to100). Table 1 is the list of diagnosed benign lesions and their frequency.

Table 2 is the list of diagnosed malignant lesions and their frequency. In the 23 cases with insufficient sample, the

Table 1. Benign lesions (final histological results)

\begin{tabular}{|l|c|c|}
\hline Histology & No & $\%$ \\
\hline Fibroadenoma & 88 & 49 \\
\hline Hamartoma & 10 & 6 \\
\hline Papiloma & 7 & 4 \\
\hline Complex sclerosing lesion & 6 & 3 \\
\hline Benign T Phillodes & 4 & 2 \\
\hline Adenosis & 3 & 2 \\
\hline Miofibroblastoma & 2 & 1 \\
\hline Fat necrosis & 1 & 0.6 \\
\hline Lymph node & 1 & 0.6 \\
\hline Lipoma & 1 & 0.6 \\
\hline Adenoma & 1 & 0.6 \\
\hline Mastitis linfocitaria & 1 & 0.6 \\
\hline Benign nonespecific & 54 & 30 \\
\hline
\end{tabular}


histological result was benign in $7(30 \%)$ and malignant in $16(70 \%)$.

Table 3 shows the correlation between the cytological findings and histological results after discarded the cases with insufficient sample. Considering all cases, regardless of the BI-RADS classification, analysis of the effectiveness of FNAC gave the following results: sensitivity $96 \%$, specificity $92 \%$, positive predictive value $97 \%$, negative predictive value $89 \%$, false positives $3 \%$, false negatives $11 \%$ and overall accuracy $95 \%$.

Table 4 shows the correlation between the cytological findings and histological results for masses classified as BI-RADS 3 ( $\mathrm{n}=111)$. The analysis of the effectiveness of FNAC for BI-RADS 3 masses gave the following results: sensitivity $100 \%$, specificity $98 \%$, positive predictive value $90 \%$, negative predictive value $100 \%$, false positives $10 \%$, there were no false negatives and overall accuracy $98 \%$.

Table 5 shows the correlation between the cytological findings and histological results for nodules classified as BI-RADS $4(\mathrm{n}=300) .17$ suspicious nodules with insufficient sample were not included. The analysis of the effectiveness of FNAC for BI-RADS 4 masses gave the following results: sensitivity $92 \%$, specificity $85 \%$, positive predictive value $95 \%$, negative predictive value $78 \%$, false positives $5 \%$, false negatives $21 \%$ and overall accuracy $91 \%$.

Table 2. Malignant lesions (final histological result)

\begin{tabular}{|l|c|c|}
\hline Histology & $\mathbf{N}^{\mathbf{~}}$ & $\mathbf{\%}$ \\
\hline IDC & 231 & 44 \\
\hline IDC/DCIS & 197 & 37 \\
\hline ILC & 65 & 12 \\
\hline DCIS & 28 & 5 \\
\hline IDC/ILC & 3 & 0.6 \\
\hline Others & 2 & 0.4 \\
\hline
\end{tabular}

Note: IDC=Invasive Ductal Carcinoma; DCIS=Ductal Carcinoma In Situ; ILC=Invasive Lobular Carcinoma

Table 3. Correlation between cytological results and the final histology

\begin{tabular}{|l|c|c|c|c|}
\hline \multicolumn{2}{|c|}{} & \multicolumn{2}{c|}{ Histology } & \multirow{2}{*}{ Total } \\
\cline { 3 - 5 } \multicolumn{2}{|c|}{} & M & B & \\
\hline \multirow{2}{*}{ Cytology } & P & 491 & 14 & 505 \\
\cline { 2 - 4 } Total & N & 19 & 158 & 177 \\
\hline
\end{tabular}

Note: $\mathrm{M}=$ Malignant; $\mathrm{B}=$ Benign; $\mathrm{P}=$ Positive; $\mathrm{N}=$ Negative

Table 4. Correlation between cytological results and the final histology for BI-RADS 3 masses

\begin{tabular}{|c|c|c|c|c|}
\hline & \multicolumn{2}{|c|}{ Histology } & \multirow{2}{*}{ Total } \\
\hline & & M & B & \\
\hline \multirow{2}{*}{ Cytology } & $\mathrm{P}$ & 18 & 2 & 20 \\
\hline & $\mathrm{N}$ & 0 & 91 & 91 \\
\hline \multicolumn{2}{|l|}{ Total } & 18 & 93 & 111 \\
\hline
\end{tabular}

Note: $\mathrm{M}=$ Malignant; $\mathrm{B}=$ Benign; $\mathrm{P}=$ Positive; $\mathrm{N}=$ Negative
Table 5. Correlation between cytological results and the final histology for BI-RADS 4 masses

\begin{tabular}{|l|c|c|c|c|}
\hline \multicolumn{2}{|c|}{} & \multicolumn{2}{c|}{ Histology } & \multirow{2}{*}{ Total } \\
\cline { 3 - 5 } \multicolumn{2}{c|}{} & M & B & \\
\hline \multirow{2}{*}{ Cytology } & P & 210 & 11 & 221 \\
\cline { 2 - 5 } & N & 17 & 62 & 79 \\
\hline Total & & 227 & 73 & 300 \\
\hline
\end{tabular}

Note: $\mathrm{M}=$ Malignant $\mathrm{B}=\mathrm{Benign} ; \mathrm{P}=$ Positive; $\mathrm{N}=$ Negative

Table 6. Correlation between cytological results and the final histology for BI-RADS 5 masses

\begin{tabular}{|l|c|c|c|c|}
\hline \multicolumn{2}{|c|}{} & \multicolumn{2}{c|}{ Histology } & \multirow{2}{*}{ Total } \\
\cline { 3 - 5 } \multicolumn{2}{|c|}{} & M & B & \\
\hline \multirow{2}{*}{ Cytology } & P & 263 & 1 & 264 \\
\cline { 2 - 5 } & N & 2 & 3 & 5 \\
\hline Total & & 265 & 4 & 269 \\
\hline
\end{tabular}

Note: $\mathrm{M}=$ Malignant; $\mathrm{B}=\mathrm{Benign} ; \mathrm{P}=$ Positive; $\mathrm{N}=$ Negative

Table 6 shows the correlation between the cytological findings and histological results for nodules classified as BI-RADS $5(\mathrm{n}=269) .6$ highly suggestive of malignancy masses with insufficient sample were not included. The analysis of the effectiveness of FNAC for BI-RADS 5 masses gave the following results: sensitivity $99 \%$, specificity $75 \%$, positive predictive value $99.6 \%$, negative predictive value $40 \%$, false positives $0.4 \%$, false negatives $60 \%$ and overall accuracy $99 \%$.

\section{Discussion}

After a time of high widespread of the FNAC for the diagnosis of breast lesions, now is entering into disuse due to the development of percutaneous techniques of histological diagnosis (core biopsy and vacuum-assisted biopsy).

However, we believe that the technique still has its place in the strategy for the diagnosis of breast masses, if it is done with strict quality control and if there is a trained medical cytologist in the institution. In the scientific literature there is a wide variability in the evaluation of the diagnostic value of FNAC. Yu and colleagues [1] conducted a metaanalysis of 46 studies including 16,642 patients with breast lesions in which FNAC was used. The sensitivity was $92.7 \%$ (95\% confidence interval, 92.1-93.3) and the specificity was $94.8 \%$ (95\% confidence interval, 94.3 95.2).

Wesola et al. [3] conducted a literature review and found sensitivity values of ultrasound-guided FNAC between $25 \%$ and $95 \%$; specificity values ranged from $97 \%$ to $100 \%$ false positives were between $1.4 \%$ and $1.6 \%$ false negatives were between $6 \%$ and $11 \%$. He et al. [4] gather 1238 patients with FNAC. The sensitivity was $87.7 \%$, specificity $99.4 \%$, false positives $0.6 \%$, false negatives $2.3 \%$ and overall accuracy $99.4 \%$.

Aker et al. [5] in a series of 733 cases of ultrasoundguided FNAC published the following results: sensitivity $98 \%$, specificity $90 \%$, overall accuracy $96 \%$, and positive 
predictive value $96 \%$, negative predictive value $94 \%$, false positive $2.6 \%$, and false negative $1.4 \%$.

The Japanese Society of Clinical Cytology published an evaluation of the results of FNAC in breast lesions in Japan [6]. 10890 patients who underwent a FNAC for a breast lesion were included. The results were the following: sensitivity $96.7 \%$, specificity $84.3 \%$, positive predictive value $92.4 \%$, false negatives $3.3 \%$, false positives $0.25 \%$ overall accuracy $88 \%$, and insufficient samples $17.7 \%$. The FNAC false positives are reported between $0.3 \%$ and $11 \%$ [7], while the false negatives between $6 \%$ and $11 \%$ $[8,9]$.

Leconte et al. [10] presented a series of 427 masses with the diagnosis of fibroadenoma after FNAC. No cancer was detected in this group, by biopsy or by follow up. The conclusion is that masses with cytological diagnosis of fibroadenoma do not require short-term follow up. Routinary follow-up is sufficient for these lesions.

Gordon et al. [11] published a study of 1070 breast masses with the cytological diagnosis of fibroadenoma. After a 3 year follow-up no cancer was diagnosed in this population. In 194 cases there was a growth of the lesion during the follow-up period (average of $20 \%$ in 6 months). The conclusion was that solid masses with benign cytological diagnosis can be followed safely and there is an acceptable growth rate of $20 \%$ for an interval of 6 months.

The wide variability of the results may be due to differences in the study design. In some of them, palpable and non-palpable lesions are included, while in others only palpable lesions; when no palpable lesions are included, in some studies stereotactic guidance is used, while in others ultrasound guidance is used or both techniques are used; in some works the cystic lesions are excluded and not in others; some studies excluded the insufficient samples from the analysis and in others they are included.

Several studies included in the analysis the cases with insufficient samples. Yu et al. [1], in their meta-analysis analyzed the effectiveness of FNAC in 11 of these studies involving insufficient samples as positive for malignancy. The results were as follows: sensitivity $92.5 \%(95 \%$ confidence interval, 90.6-93.3), specificity 76.8\% (95\% confidence interval, 75.1-78.4).

Our study is the evaluation of ultrasound-guided FNA in solid breast masses. Insufficient samples were excluded. The results are within the range of the above-mentioned results in the international literature. If we consider all the cases, the results were: sensitivity $96 \%$, specificity $92 \%$, positive predictive value $97 \%$, negative predictive value $89 \%$ false positive rate $3 \%$ false negative rate $11 \%$, overall accuracy $95 \%$. We did not find articles with a correlation between the cytological results and the likelihood of malignancy of the lesion according to its imaging features.

We believe that this exercise is relevant as it helps to make decisions in different clinical scenarios. To determine the likelihood of malignancy of each lesion we turn to the BIRADS categorization. Under this system, in the category 3 (probably benign), the likelihood of malignancy is less than or equal to $2 \%$, in the category 4 (suspicious) the likelihood is higher than $2 \%$ but less than $95 \%$ and in the category 5 (highly suggestive of malignancy) the likelihood is equal or higher than $95 \%$.

In category 3 the main objective of FNAC should be safely rule out malignancy. According to our data this objective was met because there were no false negatives and the negative predictive value was $100 \%$. There are several situations in which the FNAC is useful in this category as an alternative to imaging follow-up: requirements of the own patient due to the anxiety for the resolution of the case, in patients with increased risk, in patients who are pregnant or are planning a pregnancy during the follow-up, when there are doubts regarding compliance of imaging follow-up, or in patients already diagnosed with another concurrent malignancy.

A benign result in this category and in these situations, avoids percutaneous biopsy or surgical biopsy and an imaging follow-up could be safely planned. In categories 4 and 5, the main objective of FNAC should be safely confirming malignancy to plan appropriate treatment. According to our data the goal was met because the positive predictive value for the category 4 was $95 \%$ and for the category 5 was $99.6 \%$. When neoadjuvant chemotherapy is required, FNAC cannot replace percutaneous biopsy, as the assessment of the complete molecular profile of the tumor is necessary.

According to our data, a benign outcome in the latter categories is not acceptable since the rate of false negatives is high $(21 \%$ in category 4 and $60 \%$ in category 5). In these situations the histological diagnosis of the lesion is required. Another important finding of our research is the high percentage of cancers diagnosed in the group of patients with insufficient FNAC samples (70\%). This shows the need for a histological diagnosis when cytological sample is insufficient.

A limitation of our study is that the effectiveness of the FNAC was not evaluated in terms of age groups, lesion size or patient's risk profile. In conclusion, our research indicates that ultrasound-guided FNAC retains its value in the strategy for the diagnosis of breast solid masses. In the probably benign masses (BI-RADS 3 ) its objective is to rule out malignancy for safely avoid biopsy and planning an imaging follow-up strategy. In the suspicious and highly suggestive of malignancy masses (BI-RADS 4 and 5) the objective is to confirm malignancy prior to definitive treatment, when neoadjuvant chemotherapy is not needed. A benign result in the BI-RADS 4 and 5 categories is not acceptable and a histological diagnosis is needed, as in cases with insufficient cytological samples. 


\section{References}

1. Yu Y, Wei W, Liu J. Diagnostic value of fine needle aspiration biopsy for breast mass: A systematic review and metaanalysis. BMC Cancer 2012; 12: 41-54.

2. D’Orsi C, Sickles E, Mendelson E, et al. BI-RADS picture imaging reporting and data system. American College of Radiology, $5^{\text {th }}$ edition. 2016.

3. Wesola M, Jelén M. The diagnostic efficiency of fine needle aspiration biopsy in breast cancers -Review. Adv Clin Exp Med 2013; 22: 887-892.

4. He Q, Fan X, Yuan T, et al. Eleven years of experience reveals that fine-needle aspiration cytology is still a useful method for preoperative diagnosis of breast carcinoma. The Breast 2007; 16: 303-306.

5. Aker F, Gümrükçü G, Onomay BC, et al. Accuracy of fineneedle aspiration cytology in the diagnosis of breast cancer a single-center retrospective study from Turkey with cytohistological correlation in 733 cases. Diagn Cytopathol 2015; 43: 978-986.

6. Yamaguchi R, Tsuchiya SI. Diagnostic accuracy of fine- needle aspiration cytology of the breast in Japan: Report from the working group on the accuracy of breast fineneedle aspiration cytology of the Japanese society of clinical cytology. Oncol Rep 2012; 28: 1606-1612.

7. Patel JJ, Gartell P, Smallwood J, et al. Fine needle aspiration cytology of breast masses, an evaluation of its accuracy and reasons for diagnosis failure. Ann R Coll Surg Engl 1987; 69: $156-159$

8. Hitchcock A, Hunt C, Locker A, et al. A one year audit of fine needle aspiration cytology for the pre-operative diagnosis of breast disease. Cytopathol 1991; 2: 167-176.

9. Park I, Ham E. Fine needle aspiration cytology of palpable breast lesions. Acta Cytol 1997; 41: 1131-1138.

10. Leconte I, Abrahama C, Galantb C, et al. Can fine needle aspiration biopsy avoid short term follow-up? Diagnostic and Interventional Imaging 2012; 93: 750-756.

11. Gordon P, Gagnos F, Lanzkowsky L. Solid breast masses diagnosed as fibroadenoma at fine-needle aspiration biopsy: Acceptable rates of growth.at long-term follow-up. Radiology 2003; 229: 234-238.

\section{Correspondence to:}

Dr. Gustavo Febles,

Breast Center for Diagnosis and Treatment

(CENDYTMA),

Spanish Association,

Jaime Zudañez 2773,

apto. 1101; CP 11300,

Montevideo,

Uruguay.

Tel: 9329844585

E-mail: febles.gustavo@gmail.com 